\title{
Calibrating a Jump-Diffusion Model of an endemic invasive: Metamodels, statistics and Qfly
}

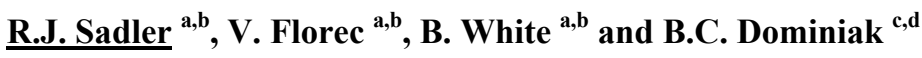 \\ ${ }^{a}$ School of Agricultural and Resource Economics, The University of Western Australia, Western Australia \\ ${ }^{b}$ Cooperative Research Centre for National Plant Biosecurity, Australian Capital Territory \\ ${ }^{c}$ Plant Biosecurity Risk Management, Industry and Investment, New South Wales \\ ${ }^{d}$ Department of Biological Sciences, Macquarie University, New South Wales \\ Email: rohan.sadler@uwa.edu.au
}

\begin{abstract}
Information on population growth and spread of a significant endemic pest such as the Queensland fruit fly (Qfly; Bactrocera tryoni) is highly valuable to biosecurity regulators wanting to evaluate the benefits of surveillance under horticultural Area Wide Management (AWM) schemes. Unfortunately, directly observing a growth model or surveillance performance is not possible due to data constraints. This paper presents a set of novel methods to infer key population and surveillance parameters from available data for a jump-diffusion model. This will form the basis of an economic analysis of AWM for the Sunraysia Pest Free Area (PFA) across south east NSW and north west Victoria.
\end{abstract}

A number of jump-diffusion models of invasive species have now been implemented to represent the different modes of dispersal available to an invasive species. However, as models become more sophisticated we encounter the perennial problem of model calibration. We implement a hierarchical model composed first of landscape 'jumps' by Qfly into the PFA, and represented by a time and spatially varying probability of declarable outbreaks derived from $\sim 150000$ monitoring events since 1998. The jumps are climate driven, with a set of time-varying Climex indices of climate, and dependent on land use, road density and other geographic factors. Here, calibration of the 'jumps' is by estimating a generalized additive model.

Assigned to each outbreak incident is an outbreak 'duration'. This duration attribute is of key interest as it will largely determine variable costs in a proposed Benefit Cost Analysis (BCA) of the surveillance methodology and new technologies. Early detection is often associated with smaller populations, lower control costs and more rapid control, and hence a decreased duration. The duration attribute is thus modeled directly as a function of the number of Qfly initially captured. Initial captures can in turn be modeled through an integro-difference model of local population growth and diffusion. However, (i) the initial location of outbreaks are unknown; (ii) the probability of detection by the monitoring network needs to be estimated; (iii) the local landscape is assumed homogeneous in the absence of further information; and, (iv) local population growth and dispersal are known to be time-varying. Ad hoc calibration methods such as least squares optimization or regression modelling were used to derive both a time invariant, half Cauchy dispersal kernel and the probability of detecting a local Qfly population, by drawing on results from the published literature. Both of these ad hoc calibrations suffer from significant bias which in future only spatially referenced and time stamped capture data can resolve.

Population growth parameters for the integro-difference model were generated from an existing stage-structured and spatially non-explicit model of Qfly population dynamics. A metamodel enabled these growth parameters to be linked to the weekly Climex indices. A time-varying distribution of initial population numbers at time of arrival (i.e. before detection), and a consequent distribution for the time to population detection, could then be simulated.

The full procedure allows elements of a monitoring regime, such as the detection efficiency of individual traps or trap density, to be economically valued in future work. While some of the calibrations in this paper are data constrained, data needs for implementation of integro-difference models in the economic valuation of monitoring networks are clear: georeferencing and time stamping of captures, experimental controls for determining growth and mortality, and ease of accessibility to such data to facilitate ready modeling.

Keywords: $\quad$ Queensland fruit fly, surveillance, detection time, area wide management 


\section{INTRODUCTION}

Benefit-Cost Analyses (BCA) of area wide management schemes (AWM) for Qfly have regularly been undertaken on a regular basis to justify public expenditure on AWM schemes such as the Sunraysia pest free area (PFA; PricewaterhouseCoopers 2001; Ha et al. 2010). However, none attempt to represent the underlying processes of 'propagule rain' of the Queensland fruit fly (Qfly; Bactrocera tryoni) into the PFA and wider Fruit Fly Exclusion Zone (FFEZ) from endemic populations in neighbouring regions. Such processes can be described as jump-diffusion (Shigesada et al. 1995), where an invasive organism has more than one mode of dispersal requiring separate process descriptions, or where dispersal occurs at both large (jump) and local (diffusion) geographical scales.

Once a Qfly outbreak occurs within the FFEZ then producers lose access to certain restricted markets (e.g. Japan) unless they apply post-harvest treatments. Rules defining an outbreak are the result of negotiated agreements among trade partners, more so than by explicit reference to the landscape ecology of the Qfly. One market rule, of monitoring weekly on a $400 \mathrm{~m}$ grid in towns and a $1000 \mathrm{~m}$ grid in production areas, incurs significant infrastructure and labour costs. The market rule thus defines a monitoring regime that is agreed by trading partners to be 'biosecure'. While economic efficiencies may be gained by varying the monitoring regime (e.g. by varying the monitoring grid), an explicit linkage of the economic benefits of monitoring to a reduced ecological risk of undetected outbreaks is generally not made within BCAs.

One mantra of monitoring is that earlier detection leads to reduced control costs later on, as at the time of detection invasive populations are likely smaller and easier to eliminate. A manager would thus seek to invest in monitoring to the extent where the marginal cost of monitoring equals the marginal benefit in saving later control costs (Kompas \& Che 2009). Time to detection, or how a jump-diffusion process interacts with a monitoring scheme, should therefore be modelled explicitly. We describe in Section 2 a probabilistic 'jump' of Qfly into the FFEZ area that is climate dependent, and model the diffusion element using a spatially and temporally explicit integro-diffusion model at the local scale (Kot \& Schaffer 1986). While the jump process can be said to be well defined from the available monitoring data, the diffusion process is not, and requires calibrations that are the focus of Section 3. Much of the interaction between monitoring and ecological process occurs at the diffusion scale in terms of the time to detection distribution. However, to value ecological risk then the jump-diffusion process needs to be simulated from a probability mechanism generating initial population sizes at different times of year (Section 4). Initial population sizes, and a given time sequence of climate, will determine both time to detection and initial population captured at the declaration of an outbreak. It is this initial capture that can be empirically related to the duration of an outbreak and the economic costs of associated market access loss. Section 5 provides concluding remarks.

\section{MODEL}

Leptokurtic (fat-tailed) distributions such as the Cauchy can mimic jump-diffusion processes, however, the jump and diffusion processes can also be modelled explicitly. For example, Carrasco et al. (2010b) provide a model of international transport, regional transport and local diffusion of the western corn rootworm, Diabrotica virgifera, with the probability of long distance jumps linked to the location of road and rail networks in Austria. For Qfly, an extensive database exists for the weekly monitoring of a grid of monitoring points across the FFEZ, with 150000 monitoring events recording 7000 capture events, leading to 141 defined outbreaks since 1998 . Road density $\left(\mathrm{km} / \mathrm{km}^{2}\right)$, land use and Climex indices of climate (e.g., cumulative drought stress and day degrees of development; Yonow \& Sutherst 1998) were found to have statistically significant relationships with the probability of outbreak using a logit link generalised additive model. Spatio-temporal autocorrelation structures can be placed on a model of the capture events, but a model translating capture events to outbreaks given some time-varying 'risk' is necessarily simple given the small number of outbreaks in total. Furthermore, this model of the 'jumps' is potentially subject to both convergence problems and the Hazuck-Donner phenomenon of large but non-significant parameter estimates due to the proportionately small number of captures relative to monitoring events (Venables \& Ripley 1999), and is at the margin of where extreme value theory may be more appropriate. The duration of outbreaks, defined as time to market recertification, is also known and has been modelled by a waiting time distribution dependent on climate and spatial variables.

While the jump process is readily modelled from the available data the local diffusion process is unobserved. Assuming a single point of arrival, a population of unknown size begins growing and spreading until contacting a sentinel monitoring point. Upon contact the population will have some probability of being detected, and in following time periods. All that is observed is the initial population captured at the declaration of the outbreak. Once the population is detected, with captures above some threshold level, an outbreak is 'declared' and eradication measures undertaken to suppress the diffusion process. 
A model of these initial population numbers for a given monitoring grid resolution provides a basis for valuing the benefits of the monitoring grid, since initial population numbers are known to influence market costs through their correlation with time to pest free recertification (i.e., outbreak duration). Integro-difference models, as spatially and temporally explicit models, can be integrated with an observation process as follows (Kot \& Schaffer 1986; Hooten \& Wikle 2008):

$$
N_{t+1}(x)=\int H_{t}\left(N_{t}(y)\right) k_{t}(x, y) d y
$$

where $N_{t}(x)$ is the population size at location $x ; H$ is a growth operator, such as the logistic growth equation $H(N)=r N(1-N / K)$ with $r$ an intrinsic growth rate and $K$ a carrying capacity; and $k$ a dispersal kernel. Both $H$ and $k$ can be time-varying (with $t$ ), spatially varying and/or stage structured functions. Computation is over a grid using fast Fourier transforms. A simple observation process can be defined for the number $z_{t}$ of Qfly captured during a sterile Qfly release-capture experiment:

$$
z_{t}\left(s \mid n_{R}, t_{R}, i\right) \sim \operatorname{Bin}\left(p_{0}, n_{t}\left(s \mid n_{R}, t_{R}, i\right)\right)
$$

where $s \in S$ is a monitoring site; $i \in I$ a release site; $n_{R}$ is the number released (at $i$ ); $t_{R}$ is a release date; and $p_{0}$ the proportion of the spatially local population $n_{t}(s)$ that is captured. In the case of a release-capture experiment then $H(N)$ is a (time-varying) mortality rate, and where there are multiple release dates and release sites then $n_{t}(s)$ is an integration of the integro-difference model over $I, T_{R}$ and $N_{R}$. Release-capture experiments can therefore supply a potential source of data for calibrating parameters of the integro-difference model.

In a purely monitoring context the key sources of uncertainty are the unknown initial population, initial location of arrival, and a stochastic detection process. The probability of detection must relate to the scale of the grid used to depict the landscape in the Fourier space computation. Further stochastic elements can be placed on either the $N_{t}(x)$ and/or $z_{t}(s)$ at each time step. By running the integro-difference model over different stochastic scenarios then a distribution of the times to detection can be derived as the time between first arrival and first detection of any given arrival, and the probability of that arrival. The key inputs of the model that require calibration before the model can be run are then: (i) weekly dispersal kernel; (ii) probability of detection; (iii) parameters of the weekly growth function; and, (iv) initial population number.

\section{DATA AND CALIBRATION}

Table 1: Matching Observed Dispersal with Distributional Percentiles (\%)

\begin{tabular}{|c|c|c|c|c|c|}
\cline { 3 - 5 } \multicolumn{2}{c}{} & \multicolumn{3}{c|}{ Dispersal Distance (m after 13 weeks) } \\
\hline Distribution & Parameter Values & Median Dispersal (m) & $\mathbf{3 0 8}$ & $\mathbf{6 0 0}$ & $\mathbf{1 0 0 0}$ \\
\hline Observed & - & - & 95.00 & 98.80 & 99.76 \\
\hline Half-Cauchy & $\lambda=2.29 ; \tau=1.01$ & 29 & 95.09 & 98.50 & 99.97 \\
\hline Normal & $\sigma=43.54$ & 105 & 95.02 & 99.98 & 100.00 \\
\hline Cauchy & scale $=24.2$ & 25 & 95.01 & 97.43 & 98.46 \\
\hline
\end{tabular}

\section{Calibration 1: Weekly Dispersal Kernel}

A dispersal kernel needs to be specified before calculating the time to detection distribution from an integrodifference model. Data are reported as distances equating to the percentile of total captures over a thirteen week period from the origin of release (Table 1; Dominiak et al. 2011). However, mark-release-capture experiments using sterile male Qfly, recording both repeated capture and release points and times (e.g., Dominiak et al. 2003), would be better suited but were unavailable. Ideally, integro-difference models would be fitted to the data directly using hierarchical Bayesian methods similar to those reported by Wikle \& Holan (2011). However, uniquely identifying dispersal, growth and probability of trap detection parameters is difficult in this context (Hooten \& Wikle 2008). Instead, percentiles of captured releases at different distances can be used to determine a weekly dispersal kernel in an ad-hoc manner similar to that of Carrasco et al. (2010a) by minimising a least squares criterion. Calculating the weekly dispersal kernel from the 13 week dispersal kernel is more difficult for the half Cauchy than for the Gaussian: unlike the Gaussian the convolution of half-Cauchy distributions does not possess an additive scale parameter, except approximately when estimating tail probabilities (Ramsay 2006). Parameters for the half Cauchy weekly dispersal kernel were searched for by convolving the dispersal kernel repeatedly, via fast Fourier transforms. The estimated half Cauchy dispersal kernel was characterised by a weekly dispersal of $9 \mathrm{~m}$ at the $95^{\text {th }}$ percentile (with median dispersal $\hat{\lambda}=2.2854$ and scale $\hat{\tau}=1.0131$ ). Note 
that leptokurtic dispersal kernels have an accelerating rate of population spread with time (Shigesada et al. 1995).

Calibration 2: Probability of Detection

Dominiak et al. (2003) report a time-varying probability of capture, ranging from $0.00004 \%$ to $0.11 \%$, across a grid of 58 monitoring points spaced $\sim 400 \mathrm{~m}$ apart following the monthly release of sterile Qfly. Confounding factors in this estimation include a time-varying mortality (related to food availability and local climate), timevarying dispersal and perhaps time-varying activity (related to temperature and describing shifting sensitivity to the cue lure traps employed). Furthermore, capture rates were reported as a proportion of the Qfly released in the previous month, and did not account for the survival of Qfly released in previous months. An assumption of a time constant probability of detection should be reasonable if the other time-varying processes can be accounted for. With only 11 monthly data points, this suggests a simple model:

$$
\ln C_{t}=\sum_{k=0}^{2} \beta_{k} R_{t-k}+s(t)
$$

where $C_{t}$ is the Poisson valued number of Qfly captures in month $t$; $R_{t}$ the number of Qfly released; and $s$ some smooth function of $t$ (here a cyclic cubic spline with few knots or degrees of freedom). Data are too few to provide the model with a useful autocorrelation structure. Constraints could be placed on the vector $\beta$, such as $\beta_{2} \leq \beta_{1}$ to reflect increasing mortality with time, and $\beta \geq 0$. However in this particular case an assumption $\beta_{1} \leq \beta_{0}$ may not hold true if an accelerating rate of population spread is assumed (i.e. few Qfly will be detected in the early weeks due to a lack of dispersal). The model can be estimated from the current data, but with so few data confidence bounds about parameter estimates will be vague, a difficulty compounded by the extremely low rate of recapture.

Estimates of $\beta_{0}$ and $\beta_{1}$ were approximately 0 , and only $\hat{\beta}_{2}=0.0001184 \%$ was statistically significant and thus retained. The time-varying smooth with four degrees of freedom shows characteristic peaks of Qfly survival and activity in late spring/early summer and autumn (Figure 1 ; adj- $R^{2}=89.3 \%$ ). The implication here is that releases two months prior to capture are more of a determining factor for capture rates than releases in either the current or previous months. This conclusion is supported in part by the weekly dispersal kernel where after two months (i.e. eight weekly convolutions) the dispersal at the $95^{\text {th }}$ percentile is $173 \mathrm{~m}$. Note that a $400 \mathrm{~m}$ resolution of the monitoring grid has average distance from the monitoring grid to a randomly located release of $\sim 140 \mathrm{~m}$. Two model assumptions can therefore be made: (i) Qfly are expiring after two months (given that further time lags $t-k(k>2)$ have zero estimates when added to the model); and, (ii) monthly capture rate per trap is $\sim 1 / 4$ of the capture rate of the entire grid of 58 monitoring points. The justification for the second assumption is that given the slow rate of dispersal and comparatively rapid mortality of individual Qfly then few of the monitoring points on a regular array, apart from the four points adjacent to a release site, will contribute much towards observed captures.

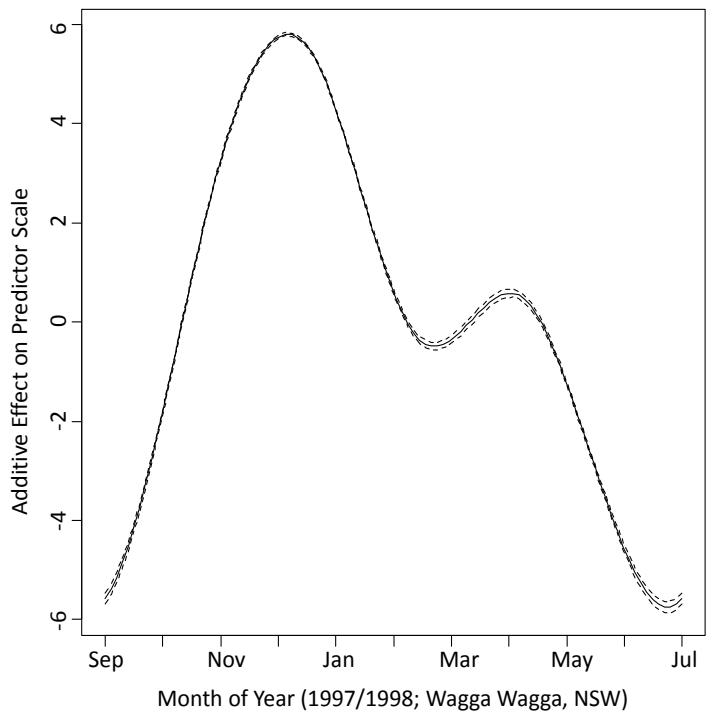

Figure 1. Time-Varying Component of $\operatorname{Pr}($ Capture)

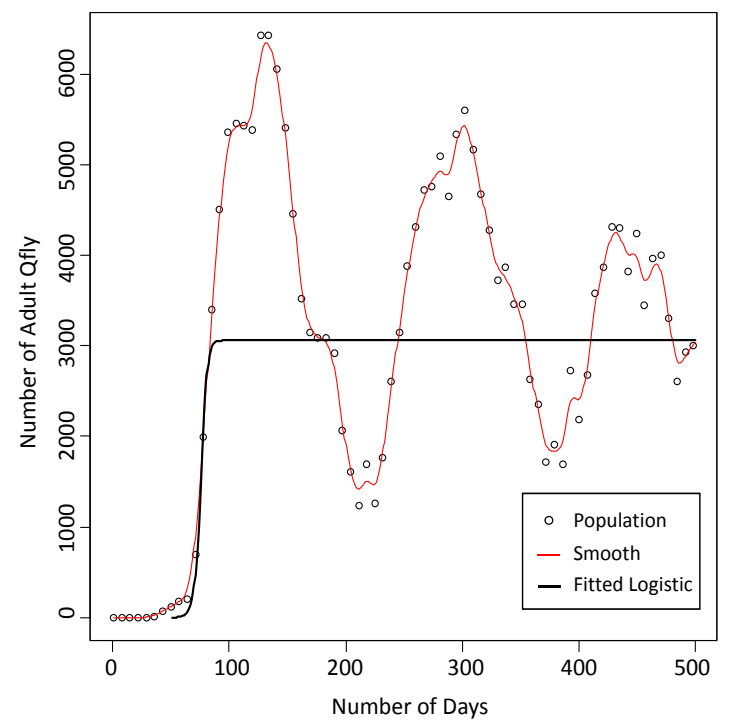

Figure 2. Estimating Logistic Growth Rates From A Stage Structured Population Model 


\section{Calibration 3: Weekly Growth Rate}

The weekly growth rate is undoubtedly time-varying, however, experimental methods for inferring these growth rates for field conditions is both difficult and costly (Sonleitner 1973), particularly over large geographical extents with varying local climes. For this purpose no suitable data exist. One feasible approach to this problem is to apply a stage structured population model calibrated for Qfly and previously implemented in the Dymex modelling environment (Yonow et al. 2004). The key driver of the population dynamics are a set of Climex derived climate indices, including a soil moisture balance and cumulative day degrees of development (Yonow \& Sutherst 1998). As we have extrapolated climate on a weekly basis over the landscape for a one arcminute resolution grid following ANUSPLIN methods (Hutchinson 1995), then growth rates can in principle be predicted at each landscape location. Growth rates for a logistic equation must first be calculated from a set of Climex indices, progressing over time since the arrival (or release) of a Qfly population. However, output from the stage structured population model returns only population numbers. Rather than filtering all the Climex indices through the population model, it would be practical to generate growth rates from the Climex indices directly. This calls for a metamodel estimated by regressing growth rates computed from the population model output on the Climex indices (Figure 2).

Growth rates and carrying capacity for a fixed set of Climex values were estimated from a long time series of adult population numbers generated by the Qfly population model (5000 days). Due to the oscillating population behavior likelihood methods for parameter estimation were not stable, and an ad-hoc approach was adopted. The carrying capacity was calculated as the long term average of population numbers, after excluding a burn-in period (1000 days). A smooth spline was then fitted to the data, and the point of maximum slope located (occurring within the first population oscillation; Figure 2). Growth rate is then the maximum slope adjusted by the carrying capacity by inverting the logistic growth equation. A 'delay' period is also estimated given that the stage structured population model exhibits a delay between egg laying and pupae emerging as adults, thereby providing a horizontal translation to the fitted logistic curve.

A quasi-Monte Carlo scheme provides a sampling design across the domain of all Climex indices (computed from Jan 1991 to Dec 2010) on which to calculate parameters of the logistic growth equation. Initial application of 10000 design points over four climate indices (max temp, shallow soil moisture, deep soil moisture and rainfall) returns adj- $R^{2}=80.7 \%$ and $89.0 \%$ for smooth tensor predictions of the growth parameter $r$ and carrying capacity $K$, when estimated independently and following stepwise model selection ( 27 and 36 degrees of freedom respectively).

\section{GENERATING TIME TO DETECTIONS AND INITIAL POPULATION SIZE}

The other key unknown variable in determining an integro-difference model for Qfly is the initial population size. A distribution of initial population numbers needs to be deduced to evaluate the risk of having an establishing population grow significantly before being detected, preferably with some dependence on climate or time of year. If the sequence of Climex indices leading up to an outbreak is known then the probability of observing a captured number can be determined by 'deconvoluting' the integro-difference model at each previous time step. This requires only the probability of detecting Qfly, a growth parameter, and dispersal kernel at any point in time, and for which the previous calibrations have been constructed.

For each time $t$ the probability of an outbreak beginning in time $F_{t}$ determines a time-varying distribution for the time to detection expected from the integro-difference model, for a specified monitoring grid (Algorithm 1). This is achieved by 'unconvolution', a likelihood inference procedure for statistically implicit models (Diggle \& Gratton 1984; Hazelton 1995), to determine the probability $P_{t, n}(Z)$ of observing captures $Z$ given arrival time $t$ and initial population size $n$. Note that the arrival time determines the historical sequence of growth parameters imputed from the metamodel and applied to the integro-difference model for each observed capture. The full empirical distribution defined by matrix $G$ is used throughout, with distributional moments such as the time-varying expected number of captures $E_{t}$ readily derived.

Now that a time-varying distribution for initial populations has been estimated, the time to detection (Error! Reference source not found.) and captured population at declaration of the outbreak can be simulated for different monitoring grid resolutions and at different times of year. These observed captures are then used to predict the duration of the loss of pest free market certification. As the costs of establishing and servicing monitoring grids of different resolutions is known then expected changes in time to detection, and variability in time to detection, can be valued when the cost of market access loss is calculated. 


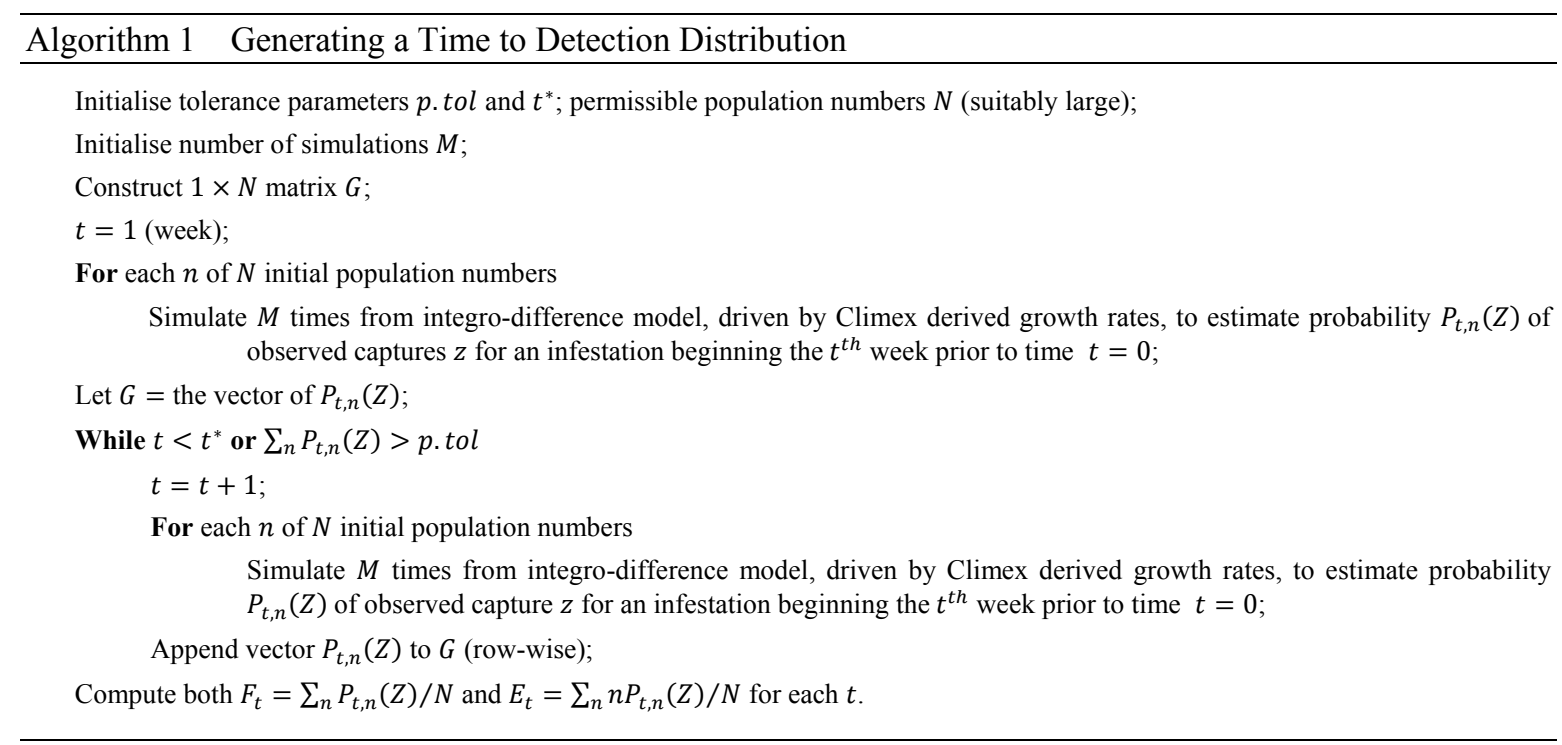

\section{CONCLUSIONS AND RECOMMENDATIONS}

1. The innovation of this paper is to provide a climate driven integrodifference model of population growth and dispersal. This model interacts with a given monitoring regime, thereby linking ecological process, and thus ecological risk, with valuing the economic benefits of monitoring.

2. The integro-difference model requires a number of calibrations, namely (i) weekly dispersal kernel; (ii) probability of detection; (iii) weekly growth parameters; and, (iv) initial population number. In this instance the first two elements were derived from data summaries reported in the existing literature, whereas the logistic growth function required a metamodel of a stage structured population model. Determination of the initial population distribution required a complex computation scheme.

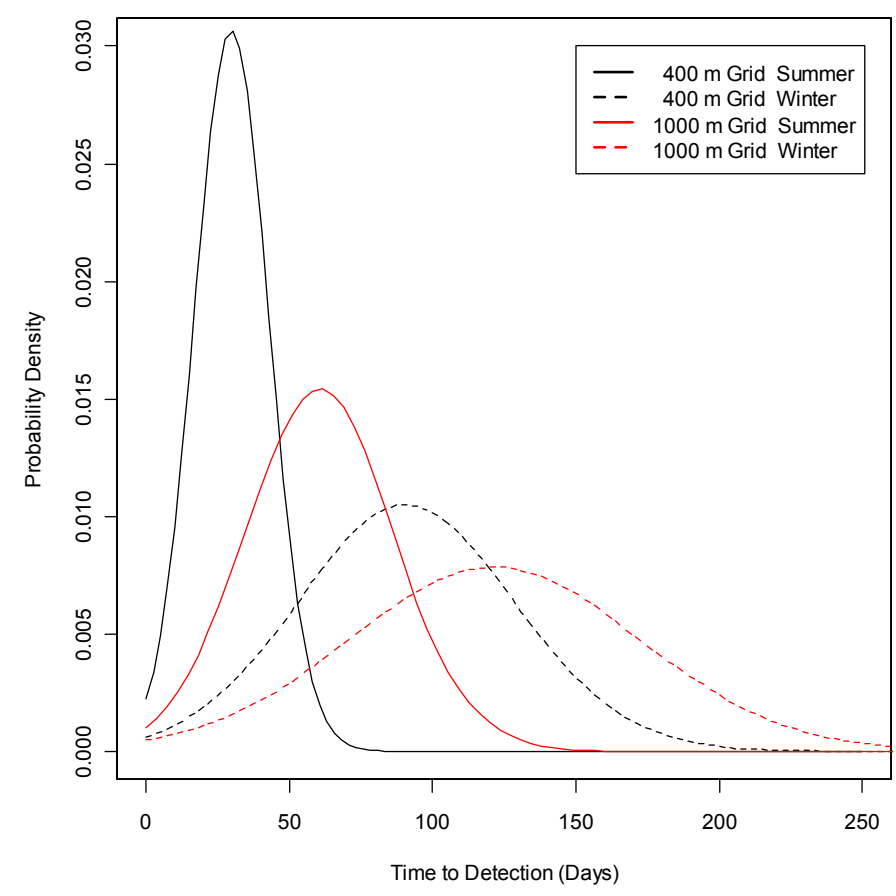

3. Calibrations 1 and 2 lack sufficient data to offer confidence intervals around the ad-hoc inferences. Furthermore, the

\section{Figure 3. Time to Detection for Different Monitoring Grids} current calibration of the dispersal kernel ignores the unobserved part of the underlying population, thereby introducing a significant overestimate of dispersal, with no captures made at the release origin. Calibrations 1 and 2 have also not been controlled for by measures of time-varying mortality rates.

4. Sterile insect release experiments offer the opportunity to estimate dispersal and trapping rates more precisely using an integro-difference model approach, for which inference procedures have recently been developed. The difficulty in such an approach will be in providing estimates of mortality, and that the probability of detection is dependent on the spatial resolution of the model. Time-varying dispersal can readily be incorporated into the integro-difference model, among other model extensions.

5. To facilitate ease of spatio-temporal modelling the biosecurity community should for maximum benefit make available sterile insect release-capture data that is both georeferenced and time stamped for each capture, and the underlying monitoring grid specified explicitly. A template for data sharing among modelers and field scientists exists in the highly successful LTER (long term ecological research) 
network in the USA, which leads to significant value-adding of already published data. Without such data availability, any calibration and consequent modeling will be severely constrained.

6. Sterile insect release-capture experiments are relatively cheap to implement in future given that existing monitoring networks in PFAs can be utilized. The difficulty will be the development of a control for mortality rates, and more generally of growth rates for wild populations. Seasonal and spatially heterogeneous food availability will likely play a key role in these population parameters. Releases could also differentiate between production areas (1000 m monitoring) where pre-harvest control is taking place and residential areas with no control $(400 \mathrm{~m})$.

\section{Acknowledgments}

The Cooperative Research Centre for National Plant Biosecurity (CRCNPB) has supported the authors in this study under Project 70100: Optimal Investment in R\&D for Plant Biosecurity. The authors would also like to thank Industry and Investment, NSW for providing the PestMon database of Qfly captures.

\section{References}

Carrasco L.R., Harwood T.D., Toepfer S., MacLeod A., Levay N., Kiss J., Baker R., Mumford J.D. \& Knight J.D. (2010a). Dispersal kernels of the invasive alien western corn rootworm and the effectiveness of buffer zones in eradication programmes in Europe. Annals of Applied Biology, 156, 63-77.

Carrasco L.R., Mumford J.D., Macleod A., Harwood T.D., Grabenweger G., Leach A.W., Knight J.D. \& Baker R. (2010b). Unveiling human-assisted dispersal mechanisms in invasive alien insects: Integration of spatial stochastic simulation and phenology models. Ecological Modelling, 221, 2068-2075.

Diggle P.J. \& Gratton R.J. (1984). Monte Carlo methods of inference for implicit statistical models. Journal of the Royal Statistical Society (B), 46, 193-227.

Dominiak B.C., Campbell A.J., Worsley P. \& Nicol H.I. (2011). Evaluation of three ground release methods for sterile Queensland fruit fly Bactrocera tryoni (Froggatt) (Diptera: Tephritidae) (in submission).

Dominiak B.C., McLeod L.J. \& Landon R. (2003). Further development of a low-cost release method for sterile Queensland fruit fly Bactrocera tryoni (Froggatt) in rural New South Wales. Australian Journal of Experimental Agriculture, 43, 407-417.

Ha A., Larson K., Harvey S., Fisher B. \& Malcolm B. (2010). Benefit-Cost Analysis of Options for Managing Queensland Fruit Fly in Victoria. Department of Primary Industries, Melbourne.

Hazelton M. (1995). Improved Monte Carlo inference for models with additive error. Statistics and Computing, 5, 343-350.

Hooten M.B. \& Wikle C.K. (2008). A hierarchical Bayesian non-linear spatio-temporal model for the spread of invasive species with application to the Eurasian Collared-Dove. Environmental and Ecological Statistics, 15, 59-70.

Hutchinson M.F. (1995). Interpolating mean rainfall using thin plate smoothing splines. International Journal of Geographical Information Systems, 9, 385-403.

Kompas T. \& Che T.N. (2009). A Practical Optimal Surveillance Measure: The Case of Papaya Fruit Fly in Australia. Australian Centre for Biosecurity and Environmental Economics, Canberra.

Kot M. \& Schaffer W.M. (1986). Discrete-time growth dispersal models. Mathematical Biosciences, 80, 109136.

PricewaterhouseCoopers (2001). Benefit Cost Analysis of the Tristate Fruit Fly Strategy. Department of Agriculture, Fisheries and Forestry, Canberra.

Ramsay C.M. (2006). The distribution of sums of certain i.i.d. Pareto variates. Communications in Statistics Theory and Methods, 35, 395-405.

Shigesada N., Kawasaki K. \& Takeda Y. (1995). Modeling stratified diffusion in biological invasions. The American Naturalist, 146, 229-251.

Sonleitner F.J. (1973). Mark-recapture estimates of overwintering survival of the Queensland fruit fly, Dacus tryoni, in field cases. Researches on Population Ecology, 14, 188-208.

Venables W.N. \& Ripley B.D. (1999). Modern Applied Statistics with S-PLUS. Springer, New York.

Wikle C.K. \& Holan S.H. (2011). Polynomial nonlinear spatio-temporal integro-difference equation models. (in submission).

Yonow T. \& Sutherst R.W. (1998). The geographical distribution of the Queensland fruit fly, Bactrocera (Dacus) tyroni, in relation to climate. Australian Journal of Agricultural Research, 49, 935-53.

Yonow T., Zalucki M.P., Sutherst R.W., Dominiak B.C., Maywald G.F., Maelzer D.A. \& Kriticos D.J. (2004). Modelling the population dynamics of the Queensland fruit fly, Bactrocera (Dacus) tryoni: a cohort-based approach incorporating the effects of weather. Ecological Modelling, 173, 9-30. 